\title{
Sex and practice distribution effects in children
}

\author{
PATRICIA A. RESICK \\ University of South Dakota, Vermillion, South Dakota 57069 \\ and \\ R. B. PAYNE \\ University of Georgia, Athens, Georgia 30602
}

\begin{abstract}
The present study, involving 40 prepubescent children in a sex by practice distribution paradigm, completed an evidentiary basis for concluding that the reversal of sex dominance in psychomotor reminiscence tendencies across pubescence was paralleled by a reversal of sex dominance in relative gains from practice distribution. These and kindred results, when viewed against those from massed practice controls, supported the inference that sex dominance in reminiscence and related tendencies, whatever the developmental phase of the subjects, occurs because males and females release reaction inhibition $\left(I_{R}\right)$ at different rates.
\end{abstract}

Several interesting dimensions have been added to the phenomenon of psychomotor reminiscence by studies that have revealed complex relationships with sex, task, and developmental factors (McCaffrey \& Payne, 1977). Especially noteworthy are those that have linked sex differences in reminiscence to an ontogenetic framework. For example, studies of young adults, using rotary pursuit and mirror tracking tasks, have shown that females reminisce more than males (Buxton \& Grant, 1939; Huang \& Payne, 1975; Payne \& Huang, 1977), but studies of prepubescent children, using the same tasks, have shown that females reminisce less than males (Horn, 1975; Zegiob \& Payne, 1977). The replicability of both findings encourages the view that sex dominance in reminiscence on these tasks, and perhaps others, is somehow reversed by factors associated with pubescence. Whatever these factors may turn out to be, it seems clear that reminiscence is far more complexly regulated than previous accounts of it have suggested.

The question naturally arises whether prevailing reminiscence theory can assimilate such results. Within the context of Hull's (1943) theory, McCaffrey and Payne (1977) proposed that a sex difference in reminiscence occurs because males and females release reactive inhibition $\left(I_{R}\right)$ at different rates. This view, of course, is compatible with $I_{R}$ theory since Hull (1945) provided for individual differences in terms of the empirical constants of equations purporting to describe behavioral laws. What is needed, however, is some kind of transsituational evidence for the generality of this proposition.

Inasmuch as $I_{R}$ theory is also basic to Hull's interpretation of distributed practice effects, it follows conjointly from this proposition and from cited data

Requests for reprints should be sent to R. B. Payne, Department of Psychology, University of Georgia, Athens, Georgia 30602. that young adult females should profit relatively more than males from practice distribution and that the sexes should exhibit essentially equal gains under a massed practice condition. In other words, the female difference between massed and distributed performances should be larger than the male difference. McCaffrey and Payne (1977) confirmed this expectation with young adult subjects in a mirror tracking situation. By the same token, of course, it follows from the work of Horn (1975) and Zegiob and Payne (1977) that prepubescent females should profit relatively less than males from practice distribution and that the sexes should exhibit essentially equal gains under a massed practice condition. These are the hypotheses to which the present work is addressed.

\section{METHOD}

\section{Subjects}

The experimental sample consisted of 40 prepubescent children ( 20 males, 20 females) recruited through churches and schools in Athens, Georgia, and surrounding communities. Ages ranged from 96 to 123 months, males averaging 111 months and females 112 months. The overall mean age was 9.3 years. All were Caucasian and right-handed.

\section{Task}

The study employed a modified version of the USAF SAM Rotary Pursuit Test, Model CM803B2, described originally by Melton (1947). The modification involved the replacement of the standard target with a laminated silver alloy target composed of a core and three concentric rings. The diameter of the core was $19 \mathrm{~mm}$, while the widths of the spacers and rings were 1.59 and $2.38 \mathrm{~mm}$, respectively. Thus, all subjects could be scored on four targets having diametric values of 19, 26.94, 34.88 , and $42.82 \mathrm{~mm}$, respectively. Rotor speed was $60 \mathrm{rpm}$. A control console provided automatic sequencing of practice periods.

\section{Procedure}

The 40 subjects were assigned without bias to two modes 
of practice distribution, except for the constraint that each mode was populated with 10 males and 10 females. One mode consisted of $2030-\mathrm{sec}$ trials of massed (continuous) practice. The other mode consisted of 2030 -sec trials separated by intertrial intervals of $30 \mathrm{sec}$. Time on target was recorded for each of the target elements in units of .01 sec.

\section{RESULTS}

\section{Performance Trends}

Inasmuch as the first trial period involved a considerable amount of interaction of experimenter and subject in the form of coaching and coaxing, we decided to eliminate Trial 1 scores from further consideration. Given this constraint, average performance trends for the four subgroups were computed for the target core and plotted as in Figure 1. Average subgroup performance trends were also computed for the larger target compounds, but they are not shown here because their inspection provides little additional information. As one might expect, performance level increased with target diameter, but the overall relationships among the subgroups showed little change from those which prevailed on the core target.

\section{Sex Effects}

Two hypotheses were examined for each of the four target diameters. The first specified that the difference between massed and distributed performance levels of males (i.e., the male mode effect) would be larger than that of females. The second specified that the sex difference in massed performance levels would not be significant in either direction, that is, both positive and negative alternatives must be ruled out.

Before proceeding to the critical analyses, one should note that the basic data were flawed in various ways. For one, the small sample size permitted a considerable amount of trial-to-trial variation, as one can see in Figure 1. For another, all trial score distributions were marked by extreme positive skewness, and the withinsubclass variances were grossly heterogeneous. The latter problems were diminished substantially by transformation of the scores to square roots, albeit at some risk to the confirmation of alternatives to the null hypothesis. All things considered, we decided further that these alternatives should be tested in a covariance paradigm in which the transformed scores from Trial 2 served as the covariate, while those from Trial 20 served as the dependent variable. Thus, the variance-covariance analyses yielded adjusted subclass means and an adjusted error term from which the standard deviation of expected replication differences could be derived for use in $t$ tests of simple effects.

With regard to performance on the target core (19 $\mathrm{mm}$ diam), the male mode effect was larger than the female mode effect $[t(35)=1.77, p<.05]$, and the sex difference in the massed mode was not significant $[t(35)=1.29, p>.05]$, as hypothesized. On the next larger target size (26.94- $\mathrm{mm}$ diam), the male mode effect was again larger than the female mode effect $[t(35)=2.28, p<.025]$, and the sex difference in the massed mode was not significant $[\mathrm{t}(35)=1.87$, $\mathrm{p}>.05]$. With regard to the third target $(34.88-\mathrm{mm}$ diam), the male mode effect continued to be larger than the female mode effect [ $t(35)=2.37, \mathrm{p}<.025$ ], but the sex difference in the massed mode was significant $[t(35)=2.07, p<.05]$, females performing better than males. On the largest target size $(42.82-\mathrm{mm}$ diam), the male mode effect was again larger than the female

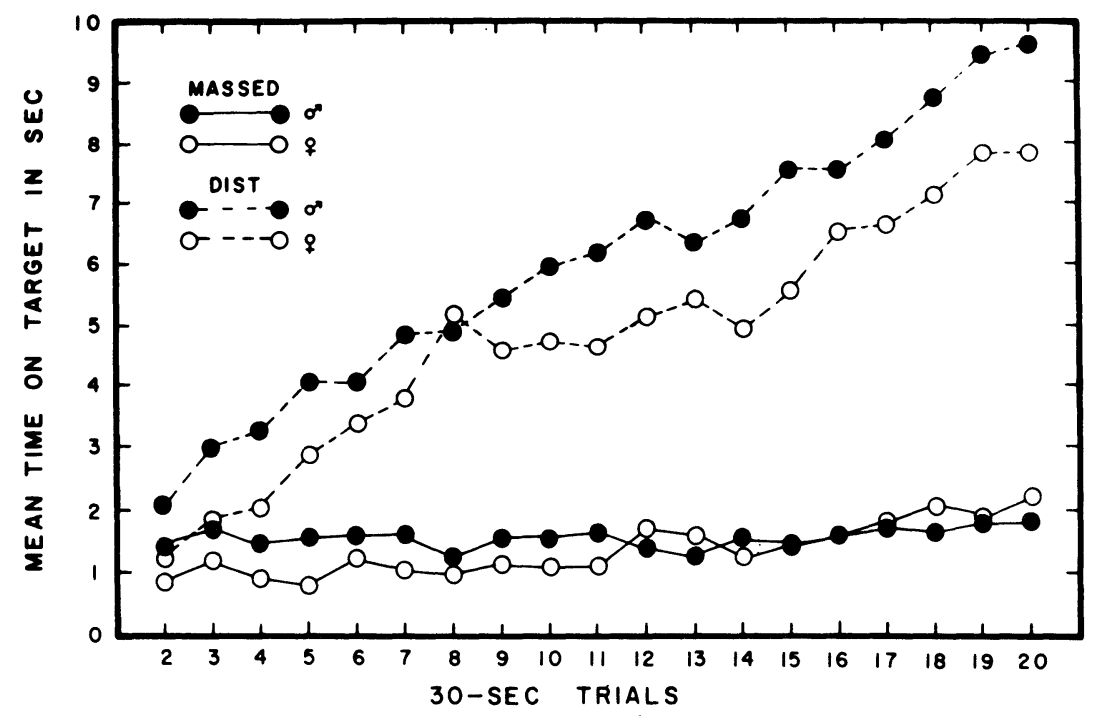

Figure 1. 
mode effect $[\mathrm{t}(35)=2.28, \mathrm{p}<.025]$, and the sex difference in the massed mode was not significant $[t(35)=1.87, p>.05]$. Thus, in three of the four target diameters the dual conditions required by theory were met in a satisfactory manner. The sex difference in the massed mode on Target 3 may have been fortuitous, but one should not overlook the possibility that there may be levels of task difficulty under which our hypothesis does not hold.

\section{Practice Distribution Effects}

It will come as no surprise that on Trial 20, and perhaps much earlier, both sexes in the distributed mode performed significantly better than their massed mode counterparts at all levels of target difficulty. The smallest $t(35)$ was $1.72, p<.05$, for females on Target 4 , while the largest $\mathrm{t}(35)$ was $5.55, \mathrm{p}<.001$, for males on Target 2. What did surprise us was the extent to which comparatively short intertrial intervals salvaged an otherwise unproductive practice regimen. We do not recall a study in which the depressant effects of massed practice upon performance were as profound as they were in the case of these young children. The relevance of this finding for the training of children in a variety of psychomotor skills is obvious.

\section{DISCUSSION}

Although the foregoing results can scarcely be described as overwhelming, they nonetheless complete an evidentiary basis for concluding that, given the tasks employed, young adult females reminisced more than males and they profited relatively more than males from practice distribution; prepubescent females reminisced less than males and they profited relatively less than males from practice distribution. Given the essential equality of the sexes and/or the absence of interaction of sex and trial effects under massed practice conditions, the results were consistent with the assumption of differential $I_{R}$ loss rates.

Our commitment to Hull's $I_{\mathbf{R}}$ principle should be regarded as nothing more than our belief that reminiscence and distributed practice phenomena are best interpreted in terms of an inhibitory factor that is generated by practice and suppresses performance unless time is provided for its spontaneous dissipation. Whatever deficiencies the rest of Hull's theory may have, the $I_{R}$ principle fulfills a persistent need, as Bilodeau and Bilodeau (1961) and Irion (1969) have pointed out. And it fulfills this need reasonably well. We acknowledge that the studies concerned with sex differences in reminiscence and distributed practice effects represent a rather trivial extension of the principle, but, at the same time, the data from such studies must be consistent with it if it is to continue as a viable hypothesis. The results of the present study are indeed consistent with this hypothesis.

What remains, of course, is the profoundly more difficult task of defining $I_{R}$ in a manner that would rationalize the demonstrable effects of various organismic variables upon reminiscence and practice distribution phenomena. Many would argue that the $I_{\mathbf{R}}$ principle was never intended to assert any functional properties of a "real" organism and that its value as a theoretical principle does not depend upon whether organismic interpretations can be found. On the other hand, reminiscence and related phenomena may be saying something very basic about neurophysiological processes involved in coping with repetitive stimulation. Moreover, the effects of such organismic variables as sex, developmental stage, excitant and depressant drugs (Hauty \& Payne, 1955), and levels of oxygenation (Hauty, Payne, \& Bauer, 1957; Dunn, Note 1) upon these behavioral phenomena constitute presumptive evidence that $I_{R}$ has organismic referents. Alas, we do not know what they are. But such data as we have presented and cited offer compelling reasons for the continued study of reminiscence and distributed practice effects in relation to various kinds of organismic manipulations.

\section{REFERENCE NOTE}

1. Dunn, J. M. Psychomotor functioning while breathing varying partial pressures of oxygen-nitrogen. (Tech. Rep. 62-82). USAF School of Aero-space Medicine, Brooks AFB, Texas, 1962.

\section{REFERENCES}

Bilodeau, E. A., \& Bilodeau, I. McD. Motor-skills learning. Annual Review of Psychology, 1961, 12. 243-280.

Buxton, C. E., \& Grant, D. A. Retroaction and gains in motor learning: II. Sex differences, and a further analysis of gains. Journal of Experimental Psychology, 1939. 25. 198-208.

Hauty, G. T., \& Payne, R. B. Mitigation of work decrement. Journal of Experimental Psychology, 1955. 49. 60-67.

Hauty, G. T., Payne, R. B., \& Bauer. R. O. Physiological costs incurred by dextro-amphetamine. Journal of Comparative and Physiological Psychology, 1957, 50. 647-651.

Horn, P. W. Pursuit rotor speed, sex differences, and reminiscence in young children. Journal of Psychology, 1975. 91. 81-85.

Huang, K. L., \& Payne, R. B. Individual and sex differences in reminiscence. Memory \& Cognition, 1975, 3. 252-256.

Hull, C. L. Principles of behavior. New York: AppletonCentury-Crofts, 1943.

Hull, C. L. The place of innate individual and species differences in a natural-science theory of behavior. Psychological Review, 1945, 52. 55-60.

IrIoN, A. L. Historical introduction. In E. A. Bilodeau (Ed.), Principles of skill acquisition. New York: Academic Press, 1969.

McCaffrey, R. J., \& PAYNe, R. B. Interaction of sex and practice distribution effects. Bulletin of the Psychonomic Society, 1977, 10, 382-384.

Melton, A. W. (Ed.). Apparatus tests. (AAF Aviation Psychology Program, Research Report No. 4) Washington, D.C: U.S Government Printing Office, 1947.

PAYNe, R. B., \& HuANG, K. L. Interaction of sex and task differences in reminiscence. Journal of Motor Behavior. 1977, 9. 29-32.

Zegiob, L., \& Payne, R. B. Reminiscence in children as a function of sex. Bulletin of the Psychonomic Society. 1977. 9, 173-175.

(Received for publication February 16, 1978.) 